EPJ Web of Conferences 77, 00004 (2014)

DOI: $10.1051 /$ epjconf / 20147700004

(C) Owned by the authors, published by EDP Sciences, 2014

\title{
Introducing Joint Research Project «Quantum Ampere» for the realisation of the new SI ampere
}

\author{
Hansjörg Scherer ${ }^{1}$, Stephen P. Giblin² ${ }^{2}$ Xavier Jehl ${ }^{3}$, Antti Manninen ${ }^{4}$, François Piquemal ${ }^{5}$, and David A. Ritchie ${ }^{6}$ \\ ${ }^{1}$ Physikalisch-Technische Bundesanstalt (PTB), Bundesallee 100, 38116 Braunschweig, Germany \\ ${ }^{2}$ National Physical Laboratory (NPL), Hampton Road, Teddington, Middlesex TW11 OLW, United Kingdom \\ ${ }^{3}$ SPSMS, UMR-E CEA/UJF-Grenoble 1, INAC, 38054 Grenoble, France \\ ${ }^{4}$ Centre for Metrology and Accreditation (MIKES), P.O. Box 9, 02151 Espoo, Finland \\ ${ }^{5}$ Laboratoire National de Métrologie et d'Essais (LNE), 29 avenue Roger Hennequin, F-78197 Trappes Cedex, France \\ ${ }^{6}$ Cavendish Laboratory, University of Cambridge, J. J. Thomson Avenue, Cambridge CB3 0HE, United Kingdom
}

\begin{abstract}
.
The metrology community lately has adopted the long-term aim of basing the SI unit system on fundamental constants of nature. The base electrical unit, the ampere, will then be re-defined in terms of a fixed value of the elementary charge $e$. The most direct realization of the new ampere definition requires controlling the number of electrons which flow in a unit time interval, and of counting the errors occurring in this process of clocking single electrons. State of the art nanofabrication technology allows the fabrication of single-electron transport devices - known as single-electron pumps - which generate electric current by moving electrons one at a time. These devices are capable of delivering currents of about $100 \mathrm{pA}$ with an accuracy at the 1 part per million level. Also, ultrasensitive single-electron detectors have been explored that allow electric charge detection on a resolution level below $e$. The European Joint Research Project presented here, undertaken by a consortium of several research institutes, aims at further developing the best existing concepts of single-electron pumps and to combine them with single-electron detectors for creating highly accurate quantum current sources, to be used as future current standards. Furthermore, necessary current measurement instrumentation will be developed. The paper comprises the project aims and the main results achieved so far.
\end{abstract}

\section{Background}

A globally consistent system of measurement units is a necessary underpinning feature of most technological, industrial and economic activities. In particular the electrical units play a vital role in the modern world where electrical measurements are all-pervasive.

The system of electrical units is grounded in the base unit ampere, whose present definition dates from 1960 and remains defined in terms of mechanical units via the laws of classical electromagnetism. Under its present classical definition, the ampere cannot be realised with an uncertainty better than a few parts in $10^{7}$, which is not sufficient to meet the accuracy needs of present and certainly future routine electrical metrology, requiring 0.1 parts per million or better. In 1990, the dilemma with the classical ampere definition led to the adoption of a practical unit system based on voltage and resistance standards - represented by the definition of "conventional values' for the von-Klitzing and Josephson constants which is decoupled from the SI.

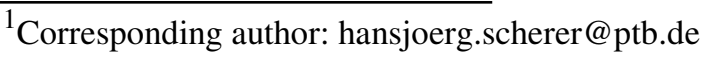

Because of these flaws, the highest global metrology authority, the Conférence Générale des Poids et Mesures (CIPM), has proposed that the ampere be re-defined in terms of the fundamental constant $e$, the charge on the electron $[1,2]$. The proposed re-definition of the ampere would restore coherence to the SI System, and would enable practical realisations of electrical units via a short, direct traceability chain in accordance with the "Mise en pratique for the ampere and other electric units in the International System of Units" [2]. The provision of a quantum-based ampere realisation at an uncertainty level of one part in $10^{7}$ is thus needed.

To realise the new ampere definition requires ways of controlling the number of electrons which flow in a unit time interval, and of counting the errors occurring in this process of clocking single electrons. State of the art nanofabrication technology allows the fabrication of single-electron transport (SET) devices - known as SET pumps - which generate electric current by moving electrons one at a time in a controllable way at sufficiently high speed and accuracy [3,4]. They provide frequency to current conversion, $I=e \cdot f$, by rapid 
controlled clocking of single electrons, and are capable of delivering currents of about $100 \mathrm{pA}$ with an accuracy at the level of one part per million. Also, ultrasensitive single-electron detectors, based on nanofabricated electrical circuits of different kinds, have been explored that allow electric charge detection on a resolution level below $e$.

\section{The JRP "Quantum Ampere"}

The Joint Research Project (JRP) Quantum Ampere is undertaken by a consortium of seven European research institutes within the frame of the European Metrology Research Programme (EMRP) [5].

Figure 1. Logo of JRP Quantum Ampere.

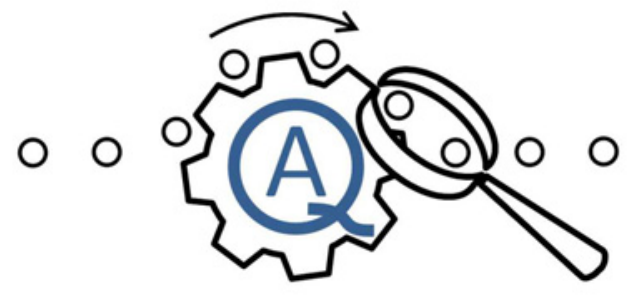

The project consortium consists of the four European National Metrology Institutes PTB (DE), LNE (FR), NPL (UK), and MIKES (FI), and is complemented by contributions from three researcher grants at CEA Grenoble (FR), University of Cambridge (UK), and Aalto University (FI). The project has started in May 2012 under coordination by PTB, and its lifetime is 36 months. Primary stakeholders of the JRP inside the metrology community are the relevant bodies and committees of the CIPM as well as National Metrology Institutes worldwide. Stakeholders outside metrology are the wider scientific community and high-level electrical instrumentation manufacturers.

\section{Scientific and technical objectives}

Recent European research work developed two promising types of single-electron current sources [3, 4]; the semiconductor tuneable-barrier pump [6-10] and the metallic hybrid turnstile [11-13], both characterised by the common feature that they can be driven by highfrequency modulation of only one single gate electrode. These so-called single-parameter devices are simpler to operate and can also achieve higher currents than previous types of SET pumps. Both device types meet the basic criteria for a metrological current standard: namely, relative ease of fabrication, ease of use, high predicted accuracy, and sufficiently high current output $I>100$ pA. Furthermore, a novel kind of SET pump based on siliconon-insulator (SOI) technology is considered a very promising candidate for a future quantum current standard [14]. The key advantage of these devices is that they are fabricated using industrial-scale CMOS techniques, opening possibilities for large-scale parallel operation at higher temperatures than other kinds of SET pumps, and integration with other electronic circuits.
To create a single-electron current standard, three main challenges remain:

1. It is necessary to verify the accuracy of the pumps at the single electron level independent of an output current measurement.

2. It is essential to develop up-scaling techniques which will enable the $100 \mathrm{pA}$ output current of the single electron devices to be amplified by a factor of 10000 or more, in order to reach practically usable current levels of the order of $1 \mu \mathrm{A}$ at an uncertainty level of the order of one part in $10^{7}$.

3. Primary current meters are needed to compare the generated currents with other reference currents, and to enable the practical use of the single-electron current sources as primary standards in a laboratory.

These challenges are addressed by the following specific scientific and technical objectives of the JRP:

- Realisation of quantum-based current sources, optimised for metrological applications with an output current of $100 \mathrm{pA}$ or higher and a relative uncertainty of 0.1 part per million or smaller;

- Development and provision of new and advanced concepts for single-electron error accounting in single-electron generating circuits by on-chip detection methods involving different kinds of singleelectron charge detectors;

- Realisation of "self-referenced" quantum current standards by integrating the above-mentioned quantum current sources and error accounting technologies;

- Realisation of quantum-based amperemeter and current amplification setups for the calibration of electrical current sources with output currents in the range of $100 \mathrm{pA}$ and for up-scaling of these currents to about $1 \mu \mathrm{A}$ for practical metrology applications.

JRP Quantum Ampere aims at further developing the best existing concepts of single-electron pumps and to combine them with single-electron detectors for creating highly accurate quantum current sources, to be used as future current standards. Furthermore, the necessary current measurement instrumentation will be developed.

All together, the project aims at providing the underpinning capabilities for the practical implementation of the Mise en pratique [2].

\section{Results and achievements so far}

\subsection{Quantum current sources and detectors}

Within the first half of project runtime, the consortium has successfully developed several kinds of SET pump devices beyond state of the art, being based on metallic (normal and superconducting) and semiconductor systems[15-21]. Some of these devices already included the single-electron detectors for monitoring charge transport at the quantum level, and first SET pump current accuracy measurements have been demonstrated by project partners PTB [15-18], NPL [10, 19], and MIKES [20]. 
At NPL and PTB pumping frequencies up to $1 \mathrm{GHz}$ have been achieved with GaAs SET pumps [10, 17, 19], and part per million accuracy could be verified for a current of $160 \mathrm{pA}$ [10]. A novel error accounting concept for SET pumps has been developed at PTB $[18,22]$. This concept suggests the use of a series combination of several SET pump devices with small intermediate "islands", their charge state being continuously monitored by single-electron detectors for detecting charge transfer errors (Figure 2, upper panel).

Figure 2. Upper panel: Detection scheme for single-electron transfer errors in series combination of several pump devices. SET transistors are used to monitor the charge state of small islands between single-electron pumps on-chip, detecting rare transfer errors [22]. Lower panel: Realization of a corresponding SET circuit consisting of four GaAs SET pumps and three intermediate Al-based SET transistors used as charge detectors [16].
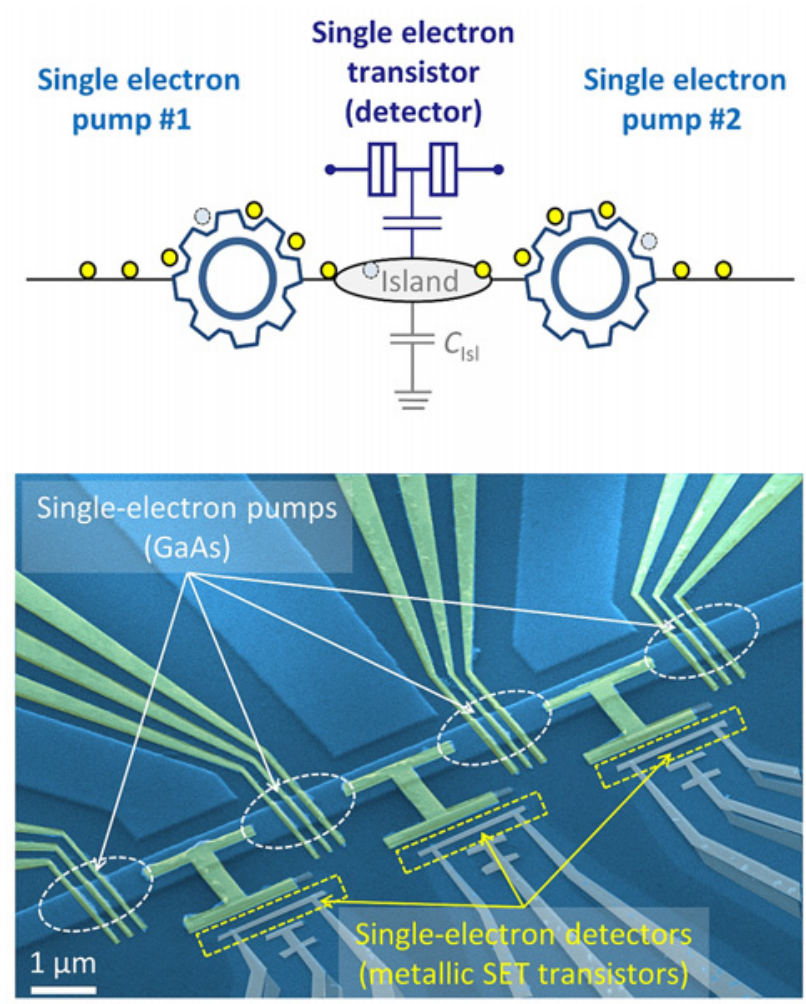

Lately, such scheme was realized at PTB, based on metallic single-electron detectors integrated in a circuit of several pumps in series (Figure 2, lower panel). First experimental results prove that this scheme for selfreferenced SET current sources is suitable for electrical quantum metrology [16].

At MIKES, a similar error detection scheme was proposed and demonstrated for hybrid turnstile devices [20]. Error mechanisms hampering the current quantization in these devices have been identified and analyzed [4].

CEA Grenoble in cooperation with LNE is developing and investigating CMOS-compatible SOI-based pump devices. A couple of devices have already been tested successfully at pumping frequencies up to $1 \mathrm{GHz}$ and up to a temperature of $0.5 \mathrm{~K}[21,23,24]$. These results confirm that SOI-based SET-pumps are promising candidates for metrology, suitable for operation at high frequencies and temperatures. Furthermore, integrated on-chip electronic circuit elements for the pump drive were fabricated and tested successfully.

\subsection{Quantum current measurement instrumentation}

High precision current measurement and up-scaling instrumentation, based on new cryogenic current comparator (CCC) systems with high winding ratios, are being developed at PTB (up to 17 000:1) [25] and at LNE (up to 30 000:1) [26-29]. These systems aim at measuring quantum generated currents of the order of $100 \mathrm{pA}$ at an accuracy level of one part in $10^{7}$. First current accuracy measurements using the CCC current amplifier setups were performed at PTB on a GaAs pump [17] and on SOI-CMOS pumps at LNE [24, 27].

As an alternative concept for current amplification, a new picoamperemeter instrument is currently under development at PTB [30]. This "Ultrastable Low-noise Current Amplifier" (ULCA) is a fully non-cryogenic single-box instrument, based on a 1000-fold current amplifier stage together with a current-to-voltage converter (transresistance amplifier) stage [31]. The effective transresistance of $1 \mathrm{G} \Omega$ is highly stable and can be calibrated traceable to a quantum Hall resistance using a CCC [25]. First tests on instrument prototypes indicate that accuracy and stability of the new amplifier type are excelling CCC-based high winding ratio current amplifiers. This will ultimately allow measurements of a $100 \mathrm{pA}$ current with a total relative uncertainty of the order of one part in $10^{7}$.

\section{Acknowledgment}

This work is supported by the European Union within the European Metrology Research Programme (EMRP). The EMRP is jointly funded by the EMRP participating countries within EURAMET and the European Union.

\section{References}

1. Draft Chapter 2 for SI Brochure, following redefinitions of the base units (2010); http://www.bipm.org/utils/common/pdf/si_brochure_ draft_ch2.pdf

2. Mise en pratique for the ampere and other electric units in the International System of Units (SI), CCEM/09-05, Note to the reader and Point 4, (2009); http://www.bipm.org/cc/CCEM/Allowed/26/CCEM09-05.pdf

3. iMERA Plus Joint Research Project "REUNIAM", Grant Agreement No. 217257, final report (2011)

4. J. P. Pekola, O.-P. Saira, V. F. Maisi et al., Rev. Mod. Phys. 85, 1421 (2013)

5. European Joint Research Project Quantum ampere: Realisation of the new SI ampere (Qu-Ampere, JRP number SIB07; http://www.ptb.de/emrp/868.html

6. M. D. Blumenthal, B. Kaestner, L. Li et al., Nat. Phys. 3, 343 (2007) 
7. B. Kaestner, V. Kashcheyevs, S. Amakawa et al., Phys. Rev. B 77, 153301 (2008)

8. B. Kaestner, V. Kashcheyevs, G. Hein et al., Appl. Phys. Lett. 92, 192106 (2008)

9. L. Fricke, F. Hohls, N. Ubbelohde et al., Phys. Rev. B 83, 193306 (2011)

10. S. P. Giblin, M. Kataoka, J.D. Fletcher et al., Nat. Comm. 3, 930 (2012)

11. J. P. Pekola, J. J. Vartiainen, M. Möttönen et al., Nat. Phys. 4, 120 (2008)

12. D. V. Averin, J. P. Pekola, Phys. Rev. Lett. 101, 066801 (2008)

13. A. Kemppinen, S. Kafanov, Y. A. Pashkin et al., Appl. Phys. Lett. 94, 172108 (2009)

14. M. Pierre, B. Roche, X. Jehl et al., in IEEE Conference Digest of the Conference on Precision Electromagnetic Measurements 2010, 755 (2010)

15. L. Fricke, M. Wulf, B. Kaestner et al., Phys. Rev. Lett. 110, 126803 (2013)

16. L. Fricke, M. Wulf, B. Kaestner et al., arXiv:1312.5669v1 (2013), to be published

17. B. Kaestner, C. Leicht, F. Hohls et al., in IEEE Conference Digest of the Conference on Precision Electromagnetic Measurements 2012, 706 (2012)

18. M. Wulf, L. Fricke, F. Hohls et al., in IEEE Conference Digest of the Conference on Precision Electromagnetic Measurements 2012, 246 (2012)

19. S. P. Giblin, M. Kataoka, J. Fletcher et al., in IEEE Conference Digest of the Conference on Precision Electromagnetic Measurements 2012, 710 (2012)

20. V. F. Maisi, O.-P. Saira, A. Kemppinen et al., in IEEE Conference Digest of the Conference on Precision Electromagnetic Measurements 2012, 248 (2012)

21. X. Jehl, B. Roche, M. Sanquer et al., in IEEE Conference Digest of the Conference on Precision Electromagnetic Measurements 2012, 250 (2012)

22. M. Wulf, Phys. Rev. B 87, 035312 (2013)

23. X. Jehl, B. Voisin, T. Charron et al., Phys. Rev. X 3, 021012 (2013)

24. X. Jehl et al., in Conference Proceedings of Métrologie 2013 (2013)

25. M. Götz, E. Pesel, D. Drung for publication in IEEE Conference Digest of the Conference on Precision Electromagnetic Measurements 2014 (2014)

26. B Steck, A. Gonzalez-Cano, N. Feltin et al., Metrologia 45, 482 (2008)

27. F. Rengnez, O. Séron, L. Devoille et al., in IEEE Conference Digest of the Conference on Precision Electromagnetic Measurements 2012, 150 (2012)

28. L. Devoille, N. Feltin, B. Steck et al., Meas. Sci. Technol. 23, 124011 (2012)

29. F. Rengnez, O. Séron, L. Devoille et al., in Conference Proceedings of Métrologie 2013 (2013)

30. H. Scherer, S. P. Giblin, X. Jehl et al., in Conference Proceedings of Métrologie 2013 (2013)

31. D. Drung, C. Krause, U. Becker et al., for publication in IEEE Conference Digest of the Conference on Precision Electromagnetic Measurements 2014 (2014) 